\title{
DISEÑO Y CONSTRUCCIÓN DE UN REACTOR DISCONTINUO CON RECIRCULACIÓN EXTERNA PARA OBTENER BIODIÉSEL A PARTIR DE ACEITE DE FRITURA EN CONDICIONES SUBCRÍTICAS
}

\author{
DESIGN AND CONSTRUCTION OF A BATCH \\ REACTOR WITH EXTERNAL RECIRCULATION \\ TO OBTAIN BIODISEL FROM RESIDUAL OIL \\ FRYING UNDER SUBCRITICAL CONDITIONS
}

\author{
Cristian Fabián Pérez-Salinas ${ }^{1, *}$, Diego Fernando Núnez-Núñez ${ }^{1}$, \\ Herminia del Rosario Sanaguano-Salguero ${ }^{2}$, Luis Fernando Sánchez-Quinchuela ${ }^{3}$
}

Recibido: 23-05-2020, Revisado: 06-08-2020, Aprobado tras revisión: 25-09-2020

\section{Resumen}

Se diseña y construye un reactor discontinuo para obtener biodiésel a partir de aceite de fritura en condiciones subcríticas con la intención de reducir el tiempo de reacción al mínimo posible. El proceso de diseño se centra en la selección del material y la verificación de su resistencia mediante un análisis FEM a partir de un diseño experimental DOE. Se consideran tres niveles de presión, temperatura y espesor de pared, respectivamente, y un factor categórico material a dos niveles. Los resultados obtenidos permiten determinar que el material apropiado para la manufactura del reactor es acero inoxidable 304 con un factor de seguridad de diseño de 1 . Para el proceso de construcción del sistema es necesario también la selección de todos los componentes complementarios. Las pruebas finales de funcionamiento muestran que es posible obtener el biocombustible en el reactor discontinuo con un grado de conversión del $88 \%$ de manera segura en un rango de 5 a 8 minutos.

Palabras clave: biodiésel, reactor discontinuo, condiciones subcríticas, DOE, elementos finitos

\section{Abstract}

A batch reactor was designed and built to obtain biodiesel from frying oil under sub-critical conditions, with the purpose of reducing the reaction time to the minimum possible. The design process is focused on the selection of the material and the verification of its resistance by means of a FEM analysis from a Design of Experiments (DOE). Three levels of pressure, temperature and wall thickness, respectively, and a material categorical factor at two levels were considered. The results obtained were that the appropriate material for manufacturing the reactor is 304 stainless steel with a design safety factor of 1 . For constructing the system it was also necessary to select all the complementary components. The final operation tests showed that it is possible to safely obtain the biofuel in the batch reactor with a degree of conversion $88 \%$, in a range of 5 to 8 minutes.

Keywords: Biodiesel, Discontinuous reactor, subcritical conditions, DOE, Finite elements.

\footnotetext{
$\overline{1, *}$ Docente investigador, Ingeniería Mecánica, Universidad Técnica de Ambato, Ecuador.

Autor para correspondencia : cf.perez@uta.edu.ec. (D) http://orcid.org/0000-0003-4031-5464

(D) http://orcid.org/0000-0001-5248-4084

${ }^{2}$ Docente investigador, Ciencias Ambientales, Universidad Estatal de Bolívar, Ecuador.

(D) http://orcid.org/0000-0002-2885-1515

${ }^{3}$ Profesor, Mecánica Industrial, Instituto Superior Tecnológico Guayaquil, Ecuador.

(D) http://orcid.org/0000-0002-4238-7376
}

Forma sugerida de citación: Pérez-Salinas, C. F.; Núnez-Núñez, D. F.; Sanaguano-Salguero, H, R. y SánchezQuinchuela, L. F. (2021). «Diseño y construcción de un reactor discontinuo con recirculación externa para obtener biodiésel a partir de aceite de fritura en condiciones subcríticas». INGENIUS. N. ${ }^{\circ} 25$, (enero-junio). pp. 32-40. DOI: https://doi.org/10.17163/ings.n25.2021.03. 


\section{Introducción}

La mayoría de los países del mundo están preocupados por reducir las emisiones de gases de efecto invernadero, aumentar las nuevas fuentes alternativas a los combustibles fósiles y recuperar el territorio deforestado con vegetación cultivable o no cultivable [1]. Para este propósito, la producción de biocombustibles es una alternativa tangible [2-4]. Se espera que el aumento en la producción de biocombustibles no solo contribuya a la conservación del medioambiente, sino también al desarrollo económico y social de los países productores [5].

Las técnicas convencionales para producir biodiésel utilizan reactores. Un reactor químico es un dispositivo complejo en el cual la transferencia de calor y masa, la difusión y la fricción pueden ocurrir junto con la reacción química bajo dispositivos de control y seguridad. Existen diferentes tipos de reactores según la forma de obtención, el tipo de flujo interno y las fases que albergan [6-9]. En general, se busca conocer el tamaño y el tipo de reactor, así como el método de operación necesario para satisfacer la obtención del producto final [10].

Uno de los parámetros importantes que se ha pretendido mejorar es el tiempo de obtención del biodiésel. En estudios previos se encuentra reportes de tiempos que van desde los 20 a los 180 minutos [11], particularmente en aceites de residuos de cocina el tiempo de obtención es de 90 minutos.

En los últimos años, la atención se ha centrado en la búsqueda de materias primas diferentes de los aceites vegetales como la soja, la palma, etc. Esto se debe principalmente al costo de obtener la materia prima, que es aproximadamente el $70 \%$ del costo total de obtener biodiésel. [6-8]. El aceite de fritura es otra fuente de materia prima, su ventaja sobre otras materias primas es que tiene la categoría de residuos. Esta característica le da una gran viabilidad porque contribuye a la reducción de la contaminación ambiental.

En Latinoamérica, el residuo de aceite de fritura tiene potencialidad de uso como fuente primaria para la producción de biodiésel [12]. Existe una industria amplia de comida rápida y tradicional que usa el aceite de fritura, pero después de su uso se convierte en un desecho. Ejemplos como en Colombia, el $35 \%$ de 162 millones de litros de la producción anual de aceite de cocina se convierte en residuo desechado en alcantarillas [13], España produce 150 millones de litros anuales de aceite vegetal usado [14].

Por otra parte, en los sectores industrial, educativo y doméstico, existen innumerables productos y sistemas desarrollados para cumplir una función específica. Todos ellos emplean materiales de ingeniería seleccionados para conferir un rendimiento óptimo. El proceso de obtención de un producto pasa por tres macroprocesos: diseño, fabricación y pruebas de fun- cionamiento. La fase de diseño comienza con la determinación de los requisitos y restricciones para llevar a cabo posteriormente un proceso de selección de material [15].

En el desarrollo ingenieril de los últimos años, se han utilizado dos métodos para la selección de materiales: el tradicional y el gráfico. El primero se basa en el conocimiento y la experiencia del ingeniero, el segundo en mapas gráficos de materiales de ingeniería organizados por sus propiedades físico-mecánicas [16].

El método gráfico se desarrolló como un soporte muy importante en la fase de concepción y desarrollo del producto $[17,18]$. El método gráfico de Michael Ashby ha tenido mayor utilidad en los últimos años. Es una metodología que sirve de guía en la selección de los materiales, contemplando los atributos que se relacionan entre sí mediante tablas de selección gráfica $[18,19]$. Se utilizan índices de rendimiento, estos se refieren a agrupaciones de las propiedades de los materiales con el fin de maximizarlos o minimizarlos de acuerdo con el requisito específico. Se derivan de la función objetivo del sistema y se expresan mediante ecuaciones matemáticas.

Varios autores informan el uso de la técnica de Análisis de Elementos Finitos (FEA) para el diseño y verificación de reactores químicos sometidos a modelado multifísico [20-23]. En estudios, como [24-26], mencionan que el diseño paramétrico proporciona a los sistemas mecánicos la posibilidad de sintetizar, simplificar y economizar el proceso de diseño, lo que permite amplificar y explorar las posibilidades de solución.

En el presente estudio, se llevó a cabo un proceso de diseño y construcción de un reactor discontinuo con recirculación externa para obtener biodiésel del aceite de fritura en condiciones subcríticas, con el propósito de reducir el tiempo de producción dentro de un marco controlado y seguro que cumpla con los requisitos de ingeniería. Para el efecto, se procedió a la selección de materiales y la verificación de la resistencia bajo el régimen de tensión utilizando la técnica de elementos finitos.

\section{Materiales y métodos}

Se siguió la siguiente metodología: selección de materiales, diseño mediante el análisis de elementos finitos (FEA), construcción del sistema y pruebas de funcionamiento. Se resalta el hecho que el diseño del reactor se inició con observación de diseños previos para concertarle en un diseño completamente nuevo que permita la reducción del tiempo de obtención del biodiésel.

\subsection{Selección de materiales}

Se utilizó el método gráfico de Ashby, con la ayuda del software educativo CES EDUPACK. Se definió la 
función requerida, el objetivo y las restricciones en el reactor (Tabla 1). A partir de la ley de Hooke que expresa la tensión en la zona elástica $(\sigma)$ es igual al módulo de Young (E) por su deformación $(\epsilon)$ y la fórmula de la masa $(\mathrm{m})$ de acuerdo con su volumen y densidad $(\rho)$, se determinó el índice de rendimiento (Ecuación 1).

Tabla 1. Definición de los requerimientos del diseño

\begin{tabular}{cc}
\hline Definición & Detalle \\
\hline Función & Alojar proceso termoquímico \\
Objetivo & $\begin{array}{c}\text { Minimizar la masa del reactor }(\mathrm{r}) \text { y maximizar } \\
\text { la resistencia mecánica }(\mathrm{s})\end{array}$ \\
& Soportar temperaturas mínimas de $160{ }^{\circ} \mathrm{C}$ \\
& y máxima de $400{ }^{\circ} \mathrm{C}$ \\
Restricciones & Soportar presión mínima de 250 psi y máxima \\
& de 3000 \\
& Resistencia a procesos oxidativo corrosivo \\
Bajo costo \\
Buena maquinabilidad y soldable
\end{tabular}

$$
\begin{aligned}
m & =\frac{F}{\epsilon \cdot E} \cdot L \cdot \rho=F \cdot L \cdot\left[\frac{\rho}{\sigma}\right] \\
& \Rightarrow \text { Indice_desempeño }=\frac{\rho}{\sigma}
\end{aligned}
$$

Tomando todas las funciones y restricciones iniciales para el reactor, se obtuvo la posibilidad de 600 alternativas de material de un total de 3900 opciones. El ajuste de la selección se realizó de acuerdo con las condiciones específicas de servicio (resistencia mecánica alta, buen comportamiento a temperatura elevadas y resistente a elementos químicos), disponibilidad local del material al menor costo posible y una densidad alta que permita una estabilidad del sistema al movimiento (Figura 1).

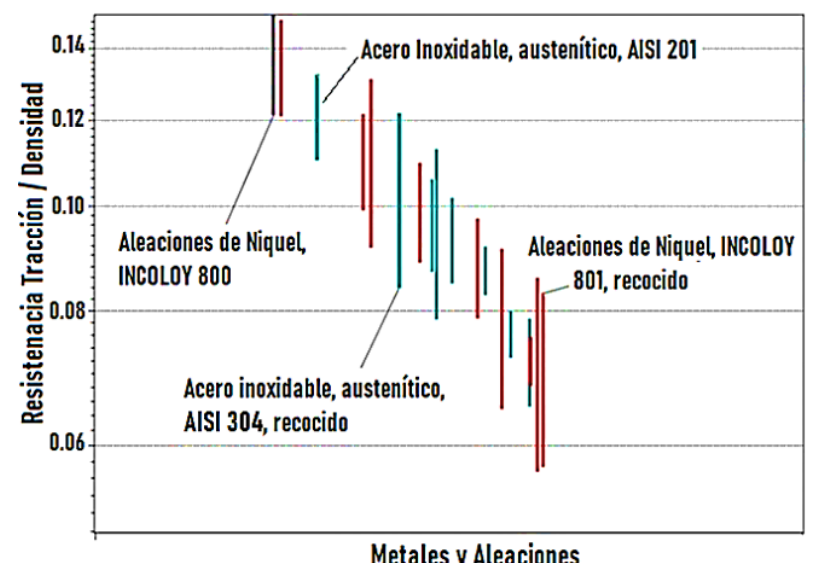

Figura 1. Gráfica de selección de materiales esfuerzodensidad [18]

Las variables de entrada fueron: precio máximo de 10 USD, densidad entre 5000 y $8000 \mathrm{~kg} / \mathrm{m}^{3}$, temperatura de servicio de $500{ }^{\circ} \mathrm{C}$, resistencia a sustancias alcalinas y sustancias con $\mathrm{pH}$ superior a 7 , capacidad de moldeo y maquinabilidad que dan como resultado 18 posibilidades de material enmarcadas en tres grupos: aceros inoxidables austeníticos, aceros inoxidables austeníticos recocidos y aleaciones de níquel.

\subsection{Análisis por elementos finitos (FEA)}

Una vez realizada la selección de materiales para el reactor, se selecciona el acero AISI 304 y la aleación de níquel para ingresar en diseño experimental FEA. Por otra parte, las dimensiones para el reactor discontinuo se plantearon para que obtenga una capacidad de recirculación continua de 1 litro.

Se planteó un diseño factorial completo donde se tomó en cuenta los tres factores principales (restricciones/requerimientos) del reactor; la presión, temperatura y espesor de pared en tres niveles respectivamente. También se consideró una variable categórica (material) en dos niveles. La Tabla 2 muestra el diseño experimental. Los rangos de los valores de presión y temperatura se basaron en estudios de obtención de biodiésel en condiciones subcríticas y supercríticas.

Tabla 2. Diseño de experimento

\begin{tabular}{cccc}
\hline \multirow{2}{*}{ Factores } & \multicolumn{3}{c}{ Niveles } \\
\cline { 2 - 4 } & Bajo & Medio & Alto \\
\hline Presión (Mpa) & 1,72 & 4,83 & 20,7 \\
Temperatura $\left({ }^{\circ} \mathrm{C}\right)$ & 160 & 200 & 400 \\
Espesor de pared (mm) & 1,5 & 2 & 3 \\
Material & Acero AISI 504 & Aleación Níquel & - \\
\hline
\end{tabular}

Se llevó a cabo un análisis termomecánico mediante elementos finitos utilizando un modelo 2D simétrico multiparamétrico realizado en Ansys APDL para evaluar la resistencia mecánica del reactor a las condiciones internas de presión y temperatura especificadas en el diseño de los experimentos. Se utilizó una cuarta parte de la sección transversal debido a los dos planos de simetría existentes en el modelo, Figura 2. Para los dos materiales utilizados en este estudio se definió un modelo isotrópico lineal elástico de material, sus propiedades se muestran en la Tabla 3.

Tabla 3. Propiedades mecánicas y térmicas para el modelo de material isotrópico elástico lineal

\begin{tabular}{ccc}
\hline \multirow{2}{*}{ Propiedades mecánicas y térmicas } & \multicolumn{2}{c}{ Material } \\
\cline { 2 - 3 } & AISI 304 & Ni 800 \\
\hline Módulo de elasticidad $(\mathrm{GPa})$ & 193 & 196 \\
Coeficiente de Poisson & 0,3 & 0,34 \\
Esfuerzo de fluencia $(\mathrm{MPa})$ & 220 & 335 \\
Densidad $\left(\mathrm{kg} / \mathrm{m}^{3}\right)$ & 7850 & 7940 \\
Temperatura de servicio $\left({ }^{\circ} \mathrm{C}\right)$ & 850 & 816 \\
Conductividad $\left(\mathrm{W} / \mathrm{kg}^{-} \mathrm{K}\right)$ & 16,2 & 11,5 \\
Calor específico $\left(\mathrm{J} / \mathrm{kg}^{\circ} \mathrm{C}\right)$ & 500 & 460 \\
Coeficiente de expansión térmica $\left(\mathrm{x} 10^{6}{ }^{\circ} \mathrm{C}^{-1}\right)$ & 17 & 12 \\
\hline
\end{tabular}




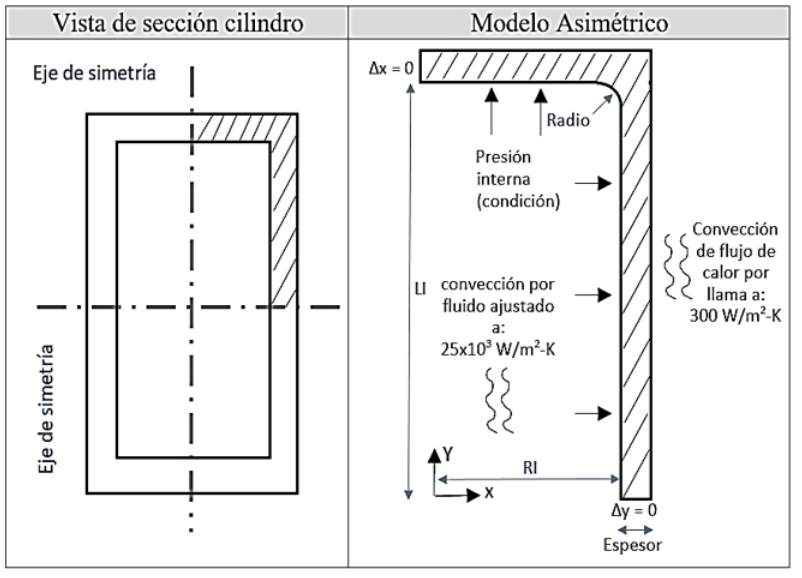

Figura 2. Geometría y condiciones límite de un modelo de reactor asimétrico de un cuarto de eje para un análisis termomecánico

El modelo se discretizó con elementos cuadriláteros PLANE182 en su versión axisimétrica; el tamaño del elemento para todos los casos se estableció en 1/5 del espesor de la pared del cilindro, ya que con este valor se aseguró una convergencia menor al $5 \%$. El modelo consta de 1830 nodos y 1520 elementos. Las condiciones límite para el análisis termomecánico estacionario se muestran en la Figura 2.

Se realizaron dos tipos de análisis de elementos finitos: uno térmico estacionario con las condiciones de temperatura y convección especificadas para obtener los desplazamientos y esfuerzos térmicos y luego un análisis estructural estático con las condiciones de frontera indicadas de tal manera que ambos estudios se acoplaron en un análisis multifísico. La validación del modelo de elementos finitos del primer tipo de análisis se realizó mediante la teoría de recipientes a presión de paredes delgadas (TWPVt) y se evaluaron las tensiones circunferenciales o tangenciales (SZ) y longitudinales o axiales (SY), porque esta teoría no tenga en cuenta las concentraciones de tensión, tales como cubiertas, agujeros o cambios abruptos en la sección transversal. En el segundo análisis de elementos finitos se evaluó la influencia de la presión y la temperatura y se compararon los resultados de la tensión de Von Misses equivalente con la tensión de fluencia de cada material para determinar el factor de seguridad del reactor.

$$
\sigma_{e}=\sqrt{\left(\sigma_{x}+\sigma_{y}\right)-3\left(\sigma_{x} \sigma_{y}-\tau_{x y}^{2}\right)}
$$

Donde:

$$
\begin{aligned}
& \sigma_{e}=\text { Esfuerzo de Von Misses } \\
& \sigma_{x}=\text { Esfuerzo normal en el eje X } \\
& \sigma_{y}=\text { Esfuerzo normal en el eje Y } \\
& \tau_{x y}^{2}=\text { Esfuerzo cortante en el plano XY }
\end{aligned}
$$

Según la teoría de los recipientes a presión de paredes delgadas (relación diámetro interno / espesor, mayor o igual a 10), existen dos tensiones principales que son función de la presión interna $(p)$, el radio interno $(r)$ y el espesor de pared $(t)$. Estas son tensiones longitudinales o axiales y circunferenciales o tangenciales, sus fórmulas de cálculo son (3) y (4) respectivamente:

Tensión longitudinal o axial (SY):

$$
\sigma_{1}=\frac{p r}{2 t}
$$

Tensión circunferencial o tangencial (SZ):

$$
\sigma_{t}=\frac{p r}{t}
$$

\subsection{Construcción del sistema}

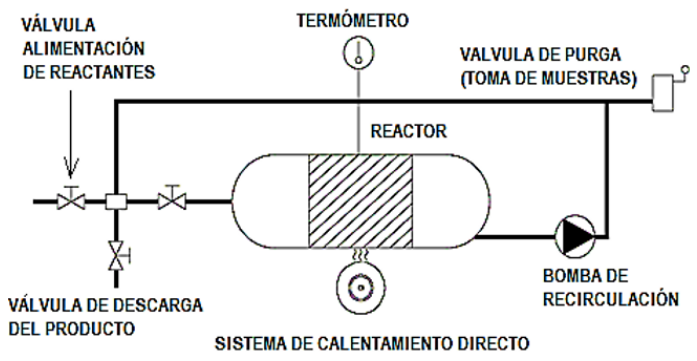

(a)

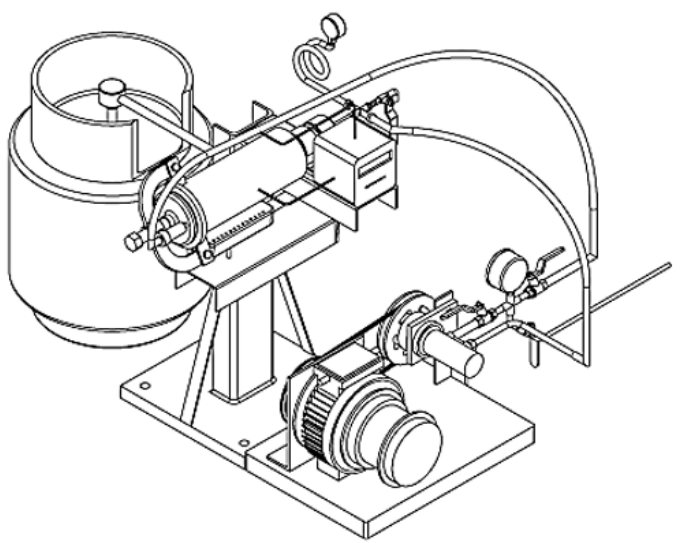

(b)

Figura 3. Reactor discontinuo a) Esquema del sistema, b) Configuración 3D

El reactor discontinuo (Figura 3) está constituido por un tanque, un tubo de circulación y una bomba de recirculación para la agitación de los reactivos. El tanque consta de un tubo cilíndrico de $280 \mathrm{~mm}$ de longitud, un diámetro interno de $71 \mathrm{~mm}$ y un espesor de pared de acuerdo con el espesor mínimo resultado del diseño (análisis FEM). El tanque está acoplado con un tubo de $1000 \mathrm{~mm}$ de largo por $12 \mathrm{~mm}$ de diámetro y una bomba de alta presión que gira a 250 rpm para recirculación y agitación. El reactor como el tubo se fabricará del material adecuado estudiado en 
el apartado 2.2 y que esté disponible al menor costo. El flujo de carga $(3 \mathrm{l} / \mathrm{min})$ es impulsado por un motor de la marca Motovario TXF005. Un termopar conectado al controlador de temperatura y un manómetro para medir la presión interna del recipiente se ubicaron en la tapa del reactor. La cubierta consta de una brida con junta de teflón. En la parte superior del reactor se colocaron dos medidores de presión Problock de 0 a 150 PSI. La temperatura del reactor es registrada por un controlador de temperatura Syscon AKC CB100.

\subsection{Pruebas de funcionamiento}

Las pruebas se llevaron a cabo a temperaturas de 160 , 180 y $200{ }^{\circ} \mathrm{C}$, con relaciones molares de metanol-aceite de $6: 1$ y $9: 1$, porcentaje de catalizador $(\mathrm{NaOH})$ de 0,5 y $1 \%$ y para un tiempo de reacción de 5 a 10 minutos para verificar la funcionalidad del reactor discontinuo con capacidad de recirculación continua de 1 litro, alcanzando presiones de 250 psi (condición subcrítica). Para la obtención del biodiésel crudo se someterá a una destilación y lavado con agua atomizada hasta que el agua de lavado se obtenga limpia. Una vez que se eliminen los productos secundarios, el biodiésel se calentará $105{ }^{\circ} \mathrm{C}$ durante 10 minutos para secar o eliminar el agua y los residuos de metanol. Una vez enfriado, se obtuvo un volumen de biodiésel refinado.

\section{Resultados y discusión}

\subsection{Resultados numéricos y analíticos}

Los resultados del análisis estructural estático se muestran en la Tabla 4. El acero inoxidable AISI 304 y la aleación de níquel Ni 800 tienen un módulo de elasticidad y una relación de Poisson muy similares, lo que significa que los resultados del esfuerzo (SY) y circunferencial (SZ) prácticamente no varían, lo que cambia es el factor de seguridad, definido como la relación entre el esfuerzo de fluencia del material y el esfuerzo equivalente máximo alcanzado. Es importante tener en cuenta que en este análisis el factor de seguridad solo se obtuvo con las tensiones circunferenciales y longitudinales, lo que no necesariamente representa un estado tensional completo del punto analizado. Según los resultados del modelo de elementos finitos, concuerda satisfactoriamente con la teoría de los recipientes a presión de pared delgada. El error máximo fue de aproximadamente $4,2 \%$.

\subsection{Influencia del reactor de presión interna y temperatura}

El estudio del acoplamiento termomecánico de una cuarta parte de la sección transversal del reactor se utilizó para determinar la influencia de la presión interna y la temperatura. Las Tablas 5, 6 y 7 muestran la influencia de la presión interna para una condición de temperatura constante de $160^{\circ} \mathrm{C}$. La leyenda del color se separó para el acero inoxidable AISI 304 y la aleación de níquel Ni800, ya que tienen un límite elástico diferente. El color rojo en la Tabla 4 significa que el material excede el límite elástico y existe la posibilidad de deformaciones permanentes que conducen a la falla del biorreactor. La escala de deformación con respecto a la condición no deformada es la misma para cada caso.

Tabla 4. Comparación de las tensiones longitudinales $\sigma_{l}(\mathrm{SY})$ y circunferenciales $\sigma_{t}(\mathrm{SZ})$ del análisis de elementos finitos (FEA) y la teoría de los recipientes a presión de pared delgada (TWPVt)

\begin{tabular}{|c|c|c|c|c|c|c|c|c|c|c|c|}
\hline $\operatorname{Exp}$ & $\begin{array}{l}\text { Presión } \\
\text { (psi) }\end{array}$ & $\begin{array}{c}\text { DOE } \\
\text { Espesor } \\
(\mathrm{mm})\end{array}$ & Material & $\begin{array}{c}\text { FEA } \\
\text { SY }\end{array}$ & $\begin{array}{c}\text { TWPVt } \\
\sigma_{l}\end{array}$ & $\begin{array}{c}\text { Error } \\
\%\end{array}$ & $\begin{array}{c}\text { Factor } \\
\text { seguridad }\end{array}$ & $\begin{array}{c}\text { FEA } \\
\text { SZ }\end{array}$ & $\begin{array}{c}\text { TWPVt } \\
\sigma_{t}\end{array}$ & $\begin{array}{c}\text { Error } \\
\%\end{array}$ & $\begin{array}{c}\text { Factor } \\
\text { seguridad }\end{array}$ \\
\hline 1 & 250 & 1,5 & AISI 304 / Ni 800 & 2857 & 2917 & 2,1 & $10,5 / 15,8$ & 5960 & 5834 & 2,1 & $5,0 / 7,6$ \\
\hline 2 & 250 & 2 & AISI 304 / Ni 800 & 2127 & 2188 & 2,8 & $14,1 / 21,2$ & 4502 & 4375 & 2,8 & $6,7 / 10,0$ \\
\hline 3 & 250 & 3 & AISI 304 / Ni 800 & 1399 & 1458 & 4,1 & $21,4 / 32,2$ & 3045 & 2917 & 4,2 & $9,9 / 14,8$ \\
\hline 4 & 700 & 1,5 & AISI 304 / Ni 800 & 8066 & 8236 & 2,1 & $3,7 / 5,6$ & 16827 & 16472 & 2,1 & $1,8 / 2,7$ \\
\hline 5 & 700 & 2 & AISI 304 / Ni 800 & 6003 & 6177 & 2,8 & $5,0 / 7,5$ & 12711 & 12354 & 2,8 & $2,4 / 3,5$ \\
\hline 6 & 700 & 3 & AISI 304 / Ni 800 & 3951 & 4118 & 4 & $7,6 / 11,4$ & 8597 & 8236 & 4,2 & $3,5 / 5,2$ \\
\hline 7 & 3000 & 1,5 & AISI 304 / Ni 800 & 34614 & 35346 & 2,1 & $0,9 / 1,3$ & 72216 & 70692 & 2,1 & $0,4 / 0,6$ \\
\hline 8 & 3000 & 2 & AISI 304 / Ni 800 & 25784 & 26510 & 2,7 & $1,2 / 1,7$ & 54552 & 53019 & 2,8 & $0,5 / 0,8$ \\
\hline 9 & 3000 & 3 & AISI 304 / Ni 800 & 16956 & 17673 & 4,1 & $1,8 / 2,7$ & 36898 & 35346 & 4,2 & $0,8 / 1,2$ \\
\hline
\end{tabular}


Tabla 5. Análisis numérico de la influencia de la presión interna del reactor

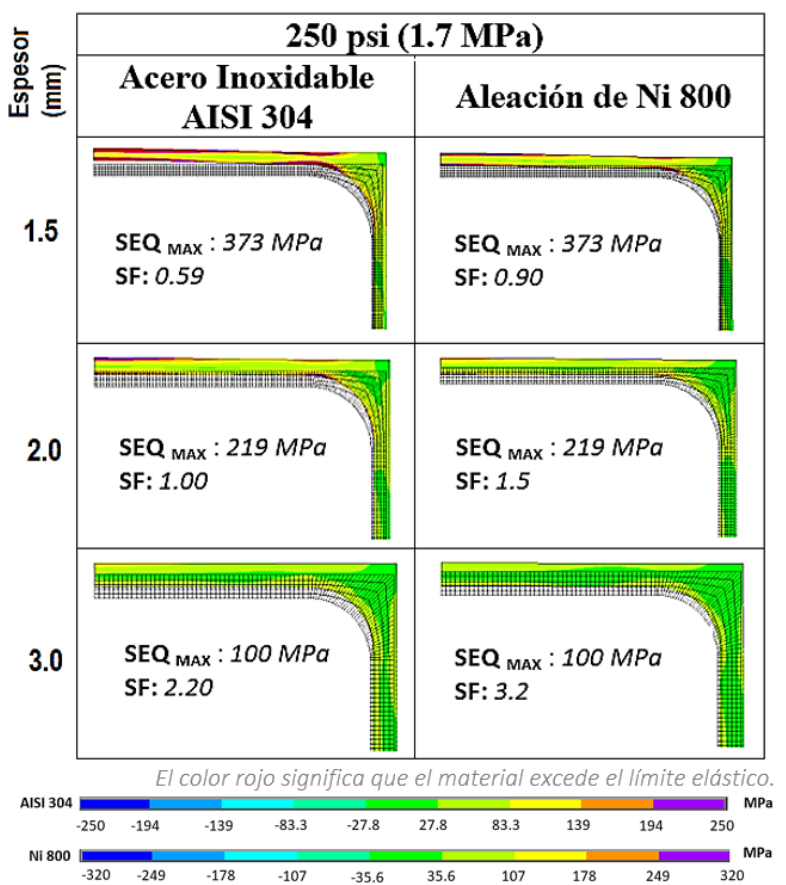

Temperatura constante $=160{ }^{\circ} \mathrm{C}$ y presión interna 250 psi (1.7 MPa)

Tabla 6. Análisis numérico de la influencia de la presión interna del reactor

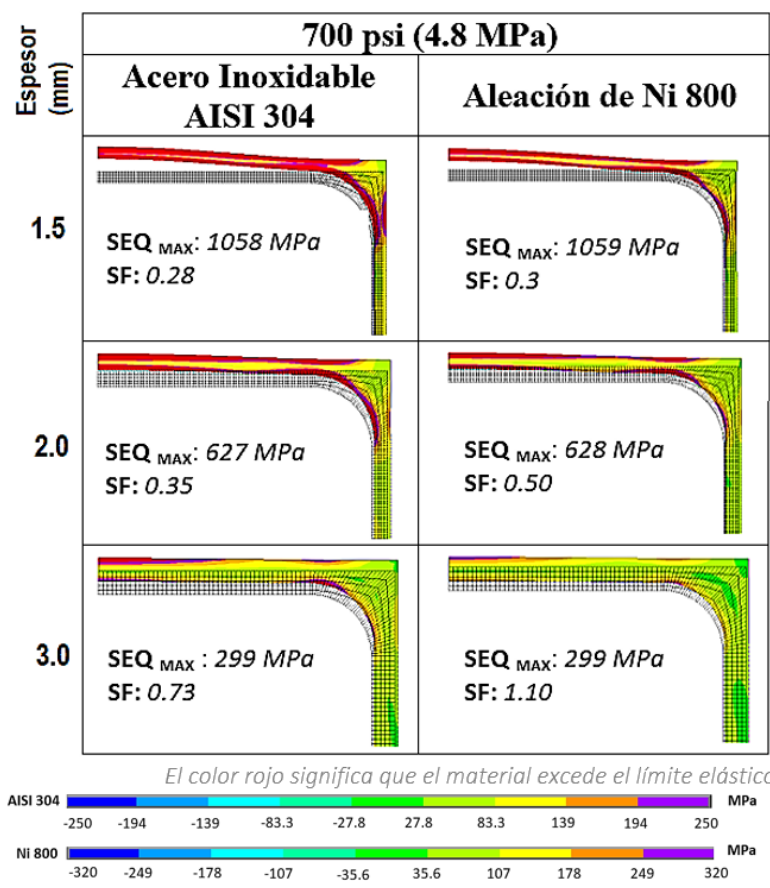

Temperatura constante $=160{ }^{\circ} \mathrm{C}$ y presión interna 700 psi (4,8 MPa)

El análisis de elementos finitos muestra resultados similares de tensión equivalente de Von Misses para ambos materiales, debido a que el módulo de elasticidad y la relación de Poisson son muy similares; sin embargo, los factores de seguridad son diferentes debido a que existe una diferencia entre su límite de elasticidad de alrededor de $100 \mathrm{MPa}$. El esfuerzo máximo de Von Misses alcanzado fue mayor para espesores de 1,5 $\mathrm{mm}$ de pared del reactor y mínimos para espesores de $3 \mathrm{~mm}$. Para una presión interna de 250, 700 y 3000 psi, el esfuerzo máximo de Von Misses fue de 373, 1058 y $4555 \mathrm{MPa}$, respectivamente.

Tabla 7. Análisis numérico de la influencia de la presión interna del reactor

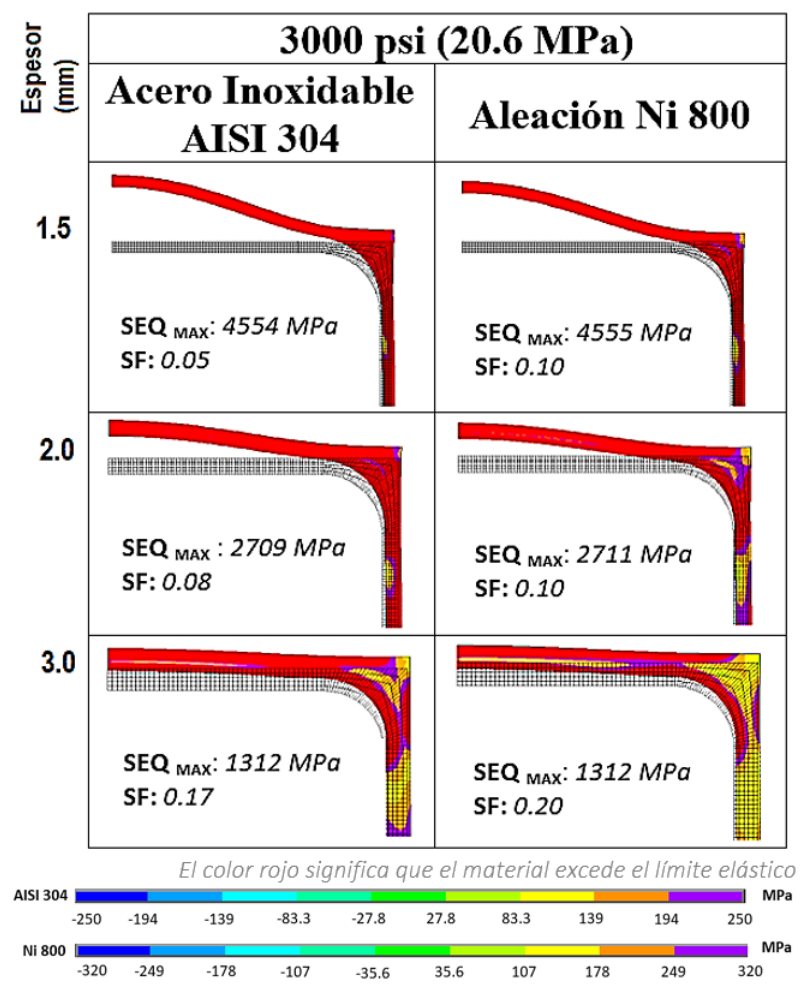

Temperatura constante $=160{ }^{\circ} \mathrm{C}$ y presión interna 3000 psi (20,6 MPa)

Con las condiciones del modelo de elementos finitos axisimétricos desarrollado en este estudio, se verifica que para el caso del acero inoxidable AISI 304 el diseño solo es seguro hasta condiciones subcríticas de presión de 250 psi y un espesor mínimo de $2 \mathrm{~mm}$ donde un factor de seguridad de 1,00 fue obtenido. El factor de seguridad se calcula como la relación entre el esfuerzo elástico del material y el esfuerzo equivalente máximo alcanzado.

Para la aleación de níquel Ni800, la presión interna se puede aumentar hasta 700 psi y un grosor de $3 \mathrm{~mm}$, donde el factor de seguridad es 1,10. Para condiciones de presión interna supercrítica (3000 psi), los factores de seguridad son inferiores a 1,00 en todos los casos, lo que indica que el material tiene una probabilidad muy alta de falla. Para esta condición, la pared de espesor del reactor debe incrementarse drásticamente. 
Las Tablas 8, 9 y 10 muestran la influencia de la temperatura para una condición de presión interna constante de 250 psi.

Tabla 8. Análisis de la influencia de la temperatura del reactor

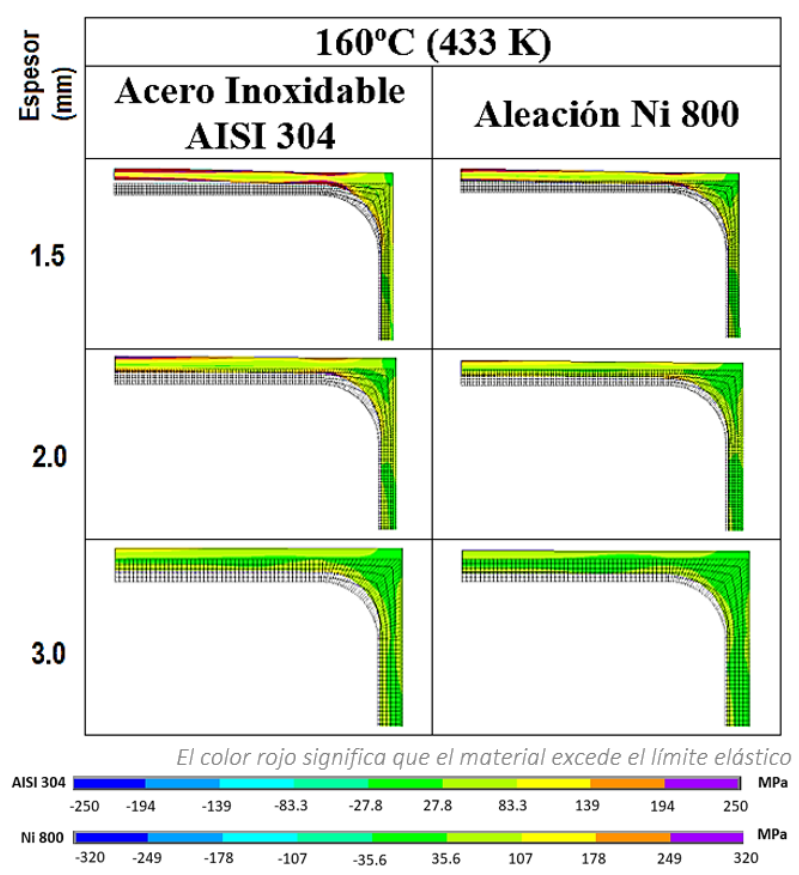

Presión constante $=250$ psi, temperaturas de $160{ }^{\circ} \mathrm{C}$

Tabla 9. Análisis de la influencia de la temperatura del reactor

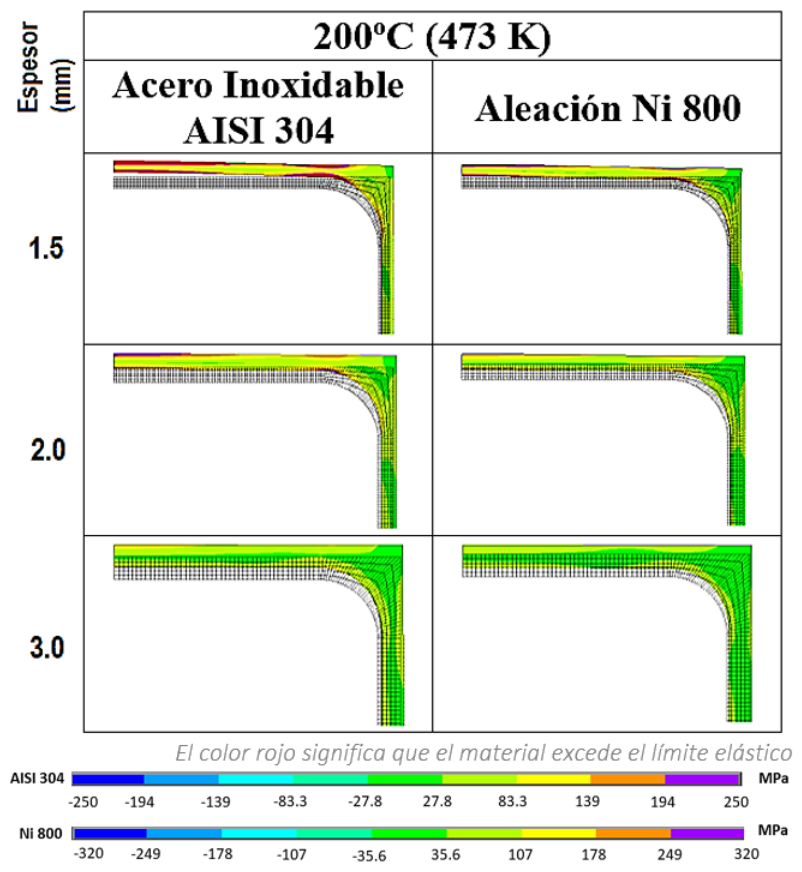

Presión constante $=250$ psi, temperaturas de $200{ }^{\circ} \mathrm{C}$
Tabla 10. Análisis de la influencia de la temperatura del reactor

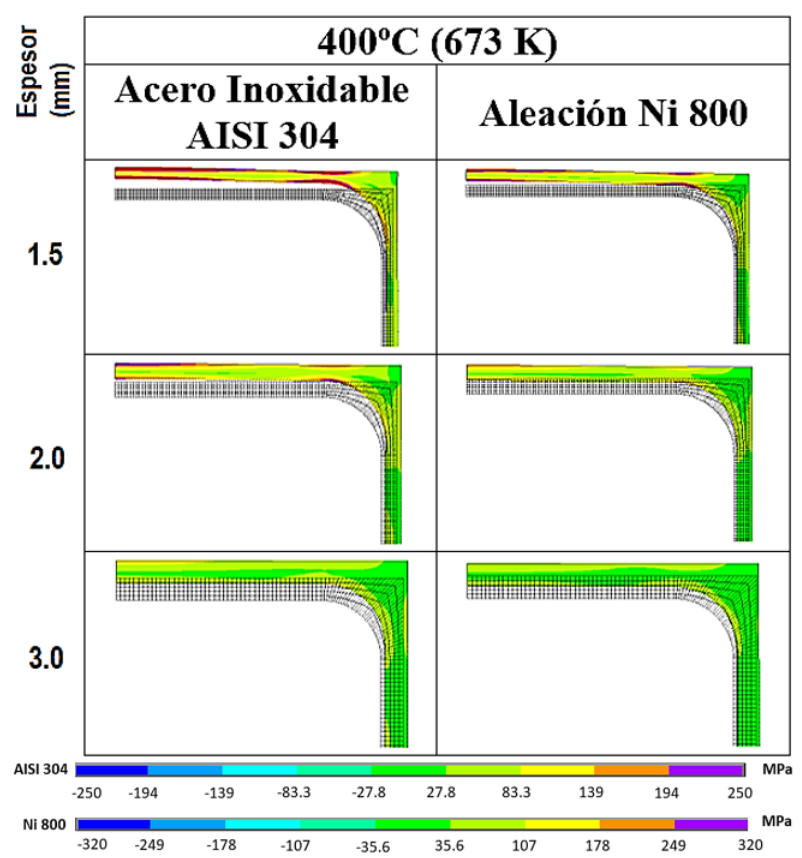

Presión constante $=250$ psi, temperaturas de $400{ }^{\circ} \mathrm{C}$

Se puede verificar que para ambos materiales las tensiones equivalentes máximas tienen una variación mínima con el aumento de temperatura 160, 200 y $400{ }^{\circ} \mathrm{C}$; por lo tanto, en este modelo los resultados solo están influenciados por el cambio en la presión interna. Se presume debido a dos factores: el gradiente de temperatura es mínimo en la pared del reactor debido a la condición estable que se alcanza con el fluido térmico interno (biodiésel) y el flujo convectivo externo (fuego). Por otro lado, ninguna de las temperaturas alcanzadas excede las temperaturas de servicio de los materiales, $850{ }^{\circ} \mathrm{C}$ en el caso del acero inoxidable AISI 304 y $816{ }^{\circ} \mathrm{C}$ en la aleación de níquel Ni800. Por lo tanto, es importante desarrollar un análisis de fatiga en una próxima investigación para estimar la vida útil del reactor.

\subsection{Pruebas de funcionamiento}

Con el prototipo desarrollado según el apartado 2.3 y las consideraciones del análisis numérico (FEM), se procedió a la realización de las pruebas de funcionamiento en laboratorio.

Una vez ejecutado el procedimiento establecido en la metodología, se obtuvo un volumen de biodiésel refinado de color amarillo transparente. Se analizaron las densidades de biodiésel (menor densidad es mejor) obtenidas tomando como referencia temperaturas de $160{ }^{\circ} \mathrm{C}, 180{ }^{\circ} \mathrm{C}$ y $200{ }^{\circ} \mathrm{C}$ utilizadas para la transesterificación (Figura 4). En la comparación de las medias de los resultados del biodiésel se encontraron diferencias estadísticamente significativas; la mayor densidad se 
dio a $200{ }^{\circ} \mathrm{C}(0,89 \mathrm{~g} / \mathrm{ml})$, mientras que a $160{ }^{\circ} \mathrm{C} \mathrm{y}$ $180{ }^{\circ} \mathrm{C}$ la densidad fue de $0,88 \mathrm{~g} / \mathrm{ml}$. El tiempo de obtención del biodiésel comienza de 5 a 10 minutos, siendo estable para obtenerlo después de 8 minutos.

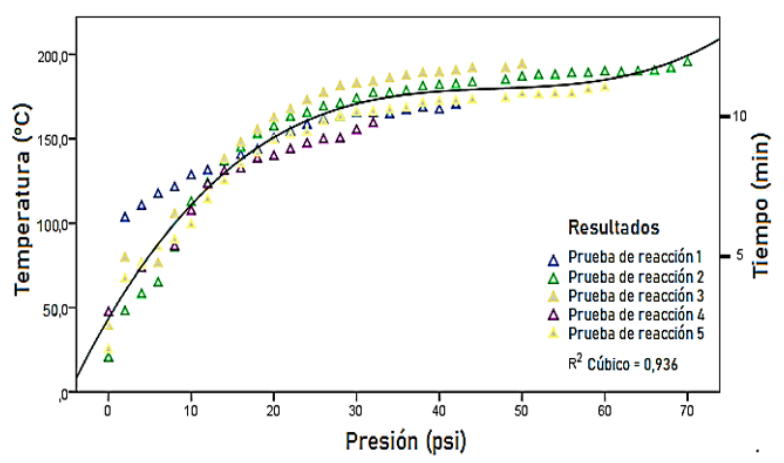

Figura 4. Comportamiento de la reacción interna del reactor en función de la temperatura, la presión y el tiempo

\section{Conclusiones}

El método gráfico de selección de material permite reducir el tiempo de selección del material. La gran cantidad de opciones para la fabricación de un reactor hace imprescindible la necesidad de un refinamiento en la selección al determinar las condiciones de servicio, tales como gradientes de temperatura, rangos de presión, ataques químicos, $\mathrm{pH}$ y consideraciones como el costo y la disponibilidad del material.

Se realizó un análisis multiparamétrico de elementos finitos simétricos en eje 2D utilizando un software Ansys APDL para evaluar la resistencia mecánica del reactor. $\mathrm{Su}$ análisis nos permite determinar la influencia de las variables en el comportamiento termomecánico del reactor. Los resultados muestran que el diseño solo es seguro hasta condiciones subcríticas de presión de 250 psi y un espesor mínimo de $2 \mathrm{~mm}$ en el caso del acero inoxidable AISI 304 donde un factor de seguridad de 1.00. En el caso de la aleación de níquel Ni800, la presión interna se puede aumentar hasta 700 psi y un espesor mínimo de $3 \mathrm{~mm}$ donde el factor de seguridad es 1.10. En las condiciones límite de este modelo, la temperatura no tiene influencia en el estrés equivalente de Von Misses.

En función del diámetro y la longitud de la sección tubular, se obtuvo un volumen de $38,48 \mathrm{~cm}^{3}$, que representa el 3,47\% del volumen total del reactor, por lo que se consideró insignificante. Por otro lado, el flujo de recirculación durante la operación del reactor manejado por la bomba es de $3 \mathrm{l} / \mathrm{min}$; en tal virtud, el fluido que pasa a través de la tubería tiene un tiempo de residencia (sección cilíndrica / flujo de la bomba), igual a $0,026 \mathrm{~min}$ (1,6 segundos), siendo evidente el efecto del comportamiento del flujo del pistón en la sección tubular, no es significativo con respecto al proceso de reacción que ocurrió en el tanque del reactor.
A través del proceso de reacción, se observó que, a mayor temperatura, más jabón se forma, lo que aumenta la densidad del biodiésel. Sin embargo, estos valores relacionados con la densidad del biodiésel obtenido en condiciones subcríticas están dentro del estándar europeo que establece un rango mínimo de $0,86 \mathrm{~g} / \mathrm{ml}$ y un máximo de $0,90 \mathrm{~g} / \mathrm{ml}$. Esta calidad de biodiésel se logró en un tiempo aproximado de 5-8 minutos.

\section{Referencias}

[1] Y. Ma and Y. Liu, "Chapter 21 - biodiesel production: Status and perspectives," in Biofuels: Alternative Feedstocks and Conversion Processes for the Production of Liquid and Gaseous Biofuels (Second Edition), second edition ed., ser. Biomass, Biofuels, Biochemicals, A. Pandey, C. Larroche, C.-G. Dussap, E. Gnansounou, S. K. Khanal, and S. Ricke, Eds. Academic Press, 2019, pp. 503-522. [Online]. Available: https: //doi.org/10.1016/B978-0-12-816856-1.00021-X

[2] L. Faba, E. Díaz, and S. Ordóñez, "Recent developments on the catalytic technologies for the transformation of biomass into biofuels: A patent survey," Renewable and Sustainable Energy Reviews, vol. 51, pp. 273-287, 2015. [Online]. Available: https://doi.org/10.1016/j.rser.2015.06.020

[3] J. Xu, G. Xiao, Y. Zhou, and J. Jiang, "Production of biofuels from high-acid-value waste oils," Energy \& Fuels, vol. 25, no. 10, pp. 4638-4642, 2011. [Online]. Available: https://doi.org/10.1021/ef2006723

[4] M. Mohammadi, G. D. Najafpour, H. Younesi, P. Lahijani, M. H. Uzir, and A. R. Mohamed, "Bioconversion of synthesis gas to second generation biofuels: A review," Renewable and Sustainable Energy Reviews, vol. 15, no. 9, pp. 4255-4273, 2011. [Online]. Available: https://doi.org/10.1016/j.rser.2011.07.124

[5] F. A. Avellaneda Vargas, "Producción y caracterización de biodiésel de palma y de aceite reclicado mediante un proceso batch y un proceso continuo con un reactor helicoidal," 2010. [Online]. Available: https://bit.ly/3jqIgdX

[6] Y. Wang, P. L. Shiyi Ou, and Z. Zhang, "Preparation of biodiesel from waste cooking oil via two-step catalyzed process," Energy Conversion and Management, vol. 48, no. 1, pp. 184-188, 2007. [Online]. Available: https://doi.org/10.1016/j.enconman.2006.04.016

[7] Y. Zhang, M. A. Dubé, D. D. McLean, and M. Kates, "Biodiesel production from waste 
cooking oil: 2. economic assessment and sensitivity analysis," Bioresource Technology, vol. 90, no. 3, pp. 229-240, 2003. [Online]. Available: https://doi.org/10.1016/S0960-8524(03)00150-0

[8] S. Zheng, M. Kates, M. Dubé, and D. McLean, "Acid-catalyzed production of biodiesel from waste frying oil," Biomass and Bioenergy, vol. 30, no. 3, pp. 267-272, 2006. [Online]. Available: https://doi.org/10.1016/j.biombioe.2005.10.004

[9] A. Srivastava and R. Prasad, "Triglyceridesbased diesel fuels," Renewable and Sustainable Energy Reviews, vol. 4, no. 2, pp. 111-133, 2000. [Online]. Available: https://doi.org/10.1016/S1364-0321(99)00013-1

[10] B. H. Pedro, A. S. John, and G. Cano, "Estudio experimental de las variables que afectan la reacción de transesterificación del aceite crudo de palma para la producción de biodiesel," Scientia et Technica, vol. 1, no. 24, ene. 2004. [Online]. Available: https://doi.org/10.22517/23447214.7323

[11] B. R. Moser, Biodiesel Production, Properties, and Feedstocks. New York, NY: Springer New York, 2011, pp. 285-347. [Online]. Available: https://doi.org/10.1007/978-1-4419-7145-6_15

[12] A. da Silva César, D. E. Werderits, G. L. de Oliveira Saraiva, and R. C. da Silva Guabiroba, "The potential of waste cooking oil as supply for the brazilian biodiesel chain," Renewable and Sustainable Energy Reviews, vol. 72, pp. 246-253, 2017. [Online]. Available: https://doi.org/10.1016/j.rser.2016.11.240

[13] A. Villabona Ortiz, R. Iriarte Pico, and C. Tejada Tovar, "Alternativas para el aprovechamiento integral de residuos grasos de procesos de fritura," Teknos revista científica, vol. 17, no. 1, pp. 21-29, jul. 2017. [Online]. Available: https://doi.org/10.25044/25392190.890

[14] Gobierno de España. (2020) Ministerio de Transición Ecológica y el Reto Demográfico. [Online]. Available: https://bit.ly/37zY23X

[15] W. D. Callister Jr. and D. G. Rethwisch, Materials science and engineering: an introduction. John Wiley \& Sons, Inc., 2018. [Online]. Available: https://bit.ly/3oo2Krl

[16] H. A. González and D. H. Mesa, "La importancia del método en la selección de materiales," Scientia
Et Technica, vol. X, pp. 175-180, 2004. [Online]. Available: https://bit.ly/37EHrMs

[17] R. V. Rao, "A material selection model using graph theory and matrix approach," Materials Science and Engineering: A, vol. 431, no. 1, pp. 248-255, 2006. [Online]. Available: https://doi.org/10.1016/j.msea.2006.06.006

[18] M. F. Ashby and K. Johnson, Materials Selection in Mechanical Design. Elsevier Ltd., 2016. [Online]. Available: https: //doi.org/10.1016/C2009-0-25539-5

[19] M. F. Ashby, H. Shercliff, and D. Cebon, Materials: engineering, science, processing and design. Butterworth-Heinemann, 2018. [Online]. Available: https://bit.ly/3dSpRW4

[20] T. E. Tezduyar and Y. J. Park, "Discontinuitycapturing finite element formulations for nonlinear convection-diffusion-reaction equations," Computer Methods in Applied Mechanics and Engineering, vol. 59, no. 3, pp. 307-325, 1986. [Online]. Available: https://doi.org/10.1016/0045-7825(86)90003-4

[21] W. F. Ramírez, Computational methods for process simulation. Butterworth-Heinemann, 1997. [Online]. Available: https://bit.ly/3mhsfc3

[22] A. Chakrabarty, S. Mannan, and T. Cagin, Multiscale Modeling for Process Safety Applications. Butterworth-Heinemann, 2015. [Online]. Available: https://bit.ly/2HujBbc

[23] W. B. J. Zimmerman, Process modelling and simulation with finite element methods. World Scientific, 2004. [Online]. Available: https://bit.ly/2G0a9vM

[24] R. Woodbury, Elements of parametric design. Routledge, 2010. [Online]. Available: https://bit.ly/2Hu2c2s

[25] R. de Luca, P. Fanelli, S. Mingozzi, G. Calabró, F. Vivio, F. Maviglia, and J. You, "Parametric design study of a substrate material for a demo sacrificial limiter," Fusion Engineering and Design, vol. 158, p. 111721, 2020. [Online]. Available: https://doi.org/10.1016/j.fusengdes.2020.111721

[26] J. Monedero, "Parametric design: a review and some experiences," Automation in Construction, vol. 9, no. 4, pp. 369-377, 2000. [Online]. Available: https://doi.org/10.1016/S0926-5805(99)00020-5 